\title{
Article \\ Symmetries of the Beijing Heping Temple Complex
}

\author{
Fan Xie ${ }^{1}$, Shuaijie Cui ${ }^{1}$ and Dongqi Sun ${ }^{2, *}$ (D) \\ 1 Faculty of Urban Construction, Beijing University of Technology, Beijing 100124, China; \\ xiefan@emails.bjut.edu.cn (F.X.); cuisj@emails.bjut.edu.cn (S.C.) \\ 2 Institute of Geographic Sciences and Natural Resources Research, Chinese Academy of Sciences, \\ Beijing 100101, China \\ * Correspondence: sundq@igsnrr.ac.cn; Tel.: +86-186-1272-9027
}

check for updates

Citation: Xie, F.; Cui, S.; Sun, D. Symmetries of the Beijing Heping Temple Complex. Symmetry 2021, 13, 1700. https://doi.org/10.3390/ sym 13091700

Academic Editors: Yeran Sun, Tomasz Lewiński, Shaohua Wang, Ting On Chan and Raffaele Barretta

Received: 22 July 2021

Accepted: 13 September 2021

Published: 15 September 2021

Publisher's Note: MDPI stays neutral with regard to jurisdictional claims in published maps and institutional affiliations.

Copyright: (c) 2021 by the authors. Licensee MDPI, Basel, Switzerland. This article is an open access article distributed under the terms and conditions of the Creative Commons Attribution (CC BY) license (https:// creativecommons.org/licenses/by/ $4.0 /)$.

\begin{abstract}
Three-dimensional laser scanning technology has been more mature, and its application fields are expanding. It is being used in key projects and important work such as ancient building recording, restoration and reconstruction. In this paper, the technology is applied to the 3D scanning, data splicing and model simplification of Heping temple building complex in Beijing. After innovative research on ancient architecture, it is found that the group layout, single form and local components of Heping temple building complex in Beijing show symmetry everywhere, vividly reflecting the extensive and profound ancient architectural culture and order. This points out a new direction for the development and application of 3D laser scanning technology and opens up a new path for the in-depth study of the protection of ancient buildings in China.
\end{abstract}

Keywords: 3D laser scanning technology; ancient architectural buildings; symmetry; point cloud model; scale analysis

\section{Introduction}

In the fields of Science and Arts, symmetry is always a topic that is worthy of studying. In Mathematics, there are mapping symmetry, translation symmetry, rotation symmetry and other forms of symmetry [1]. In Arts, there are axial symmetry, central symmetry and others [2]. These forms of symmetry can deepen the analysis of their characteristics in respective fields [3]. At the same time, research about symmetry plays an extremely important role in ancient architecture. The builders of yore used different forms of symmetry in construction, illustrating a serious and concise form of order, which is inseparable from the cultural and value attributes of ancient architecture. Chinese ancient architecture focuses on the epitome of symmetry. The most important aspect of Chinese architecture is the establishment of the main central line [4]. From the building complex itself, to individual layout, and then to local components, architects attach great importance to the placement of the central axis. Most of the ancient Chinese buildings are built with the central axis as the core [5]. Therefore, understanding symmetry is of great significance for the study of the cultural and aesthetic value of ancient construction.

In order to study the symmetry of ancient buildings more accurately, 3D laser scanning technology is used to intuitively express the analysis results through scientific means. In the 1950s, when the United States and the former Soviet Union competed for hegemony, the former Soviet Union first applied laser technology to positioning, which was the rudiment of the application of 3D laser scanning technology. From then on, the application of 3D laser scanning technology has been expanding under the development of economy, science and technology, culture and other aspects [6]. At the end of the 20th century, the application of 3D laser scanning technology in the film industry has become increasingly mature, and its application was then extended to the military industry, locomotive manufacturing and other heavy industries $[7,8]$. Two aspects of information are collected to study Chinese ancient architecture by using 3D laser scanning technology. One is Chinese ancient constructions' structure, including the specific size, length, shape, topological relationship and 
other 3D space specific information; the other is their appearance attribute information, such as color and texture. In recent years, the technology has been widely used in the research, restoration and reconstruction of ancient buildings [9,10], 3D landscape simulation of urban and rural blocks, digital museums and virtual tourism [11].

The Levoy team used industrial scanners to collect 3D data of Michelangelo's statue David [12]. Through detailed observation of the scratches, the team carried out research on Michelangelo's carving tools. The scanning team initially established a complete process for building scanning, but the hardware configuration was not ideal at that time, so the overall research only yielded a shallow understanding. In the centennial overhaul of the Forbidden City in 2002, the layout method of the control network and the digital technologies of data splicing, impurity removal and compression were studied, focusing on the 3D laser scanning technology, and the application fields were discussed [13]. W. Boehler and his research team used traditional technology and 3D laser scanning technology to study five kinds of objects, including ancient walls, relief decorative panels, stone age handicrafts, ancient statues and the external space of the ancient castle [14]. They concluded that 3D laser scanning technology and close-range photogrammetry have both advantages and disadvantages, while 3D laser scanning has great advantages in the speed of data acquisition and final accuracy. In the final precision contrasting experiment, the 3D scanner is dominant in most areas. At the same time, due to the large weight of the instrument, it is difficult to replace the traditional measurement methods.

In the restoration of Bilan Bridge in Old Summer Palace, the school of architecture of Tsinghua University analyzed various components of the bridge by using 3D laser scanning technology and simulated the restoration work on the computer [13]. During the restoration of Dazu Stone Carvings' finger, the Chinese Academy of Cultural Heritage used 3D laser scanning technology on the basis of obtaining comprehensive and accurate data. Therefore, they used a combination of virtual technology and reality to study sections, colors and other aspects of the carving [15]. Gabriele Guidi and Fabio Remondino's team used multi-resolution and multi-sensor scanning technology to scan an area of Pompeii's ancient city [16]. This research has made beneficial exploration in cross-scale, fusion of multi-precision data and 3D display of the outcomes. To a certain extent, it also improved the defects of the large amount of point cloud data and difficult of use. In Maria Giuseppa Angelini's digital acquisition research of the Chateau de Monte in Italian, the total station is used as the control network, combined with the 3D ground laser scanning, which effectively reduces the cumulative error of data splicing. The process of using Laika special data processing software CYCLONE to slice the 3D point cloud was also discussed and, finally, the two-dimensional architectural drawing was generated. This research has opened the door for the automatic generation of three-view drawings from 3D data. However, there is a huge technical gap in the automatic generation technology for the visible line part of 2D data, which limits the application of point cloud data to a certain extent [17]. In the "Technical Specification for 3D Laser Scanning on the Ground" issued by the China Bureau of Surveying, Mapping and Geographic Information, general provisions are put forward on the scope of use, the professional terms are quoted from the documents and the requirements for preparation, collection, processing and results are put forward according to the actual operation steps. The release of the regulation provides a reasonable framework for the research of 3D laser scanning technology. The "Technical Specification for 3D Information Collection of Cultural Relic Buildings" issued by Beijing puts forward and stipulates the basic technical requirements for 3D information collection of cultural relic buildings in data collection, processing and production. It provides solutions for how to collect the information of cultural relic buildings, how to set and select reasonable and effective collection accuracy and how to deal with acceptance and filing [18].

To sum up, in the application of 3D laser scanning technology, Stanford University, Politecnico di Milano and other relevant institutions have carried out different degrees of exploration and applied their findings to the protection of ancient buildings and other practical projects. However, at present, most hardware standards and operation processes 
are formulated by hardware manufacturers. The standards, parameters and processes of scanners focus on hardware equipment, and there is a lack of scanning research for the needs of ancient buildings. At the same time, the structure of Chinese ancient buildings is spliced by a mortise and tenon with a complex relationship, such as bracket set, rafter and purlin. It is complex, and each element covers on top of each other. In terms of the difficulty of data collection and the richness of collection types, it is difficult for Chinese ancient wooden buildings to ensure the integrity and authenticity of three-dimensional collection. Therefore, for the three-dimensional information collection of Chinese ancient wooden buildings, it is necessary to design technical processes and methods to ensure the overall efficiency and accuracy of collection. In addition, the splicing of point cloud model needs to convert and unify the coordinates of different shapes from multiple angles and then synthesize the multi-angle information. The more complex the shape, the more stations needed to be erected, and the 3D scanner has two scanning dead angles: the conical area under the instrument and the deep hole smaller than the size of the scanning instrument. These two dead angles appear everywhere in ancient buildings, making it difficult to determine their authenticity. At present, the research on this technology, mostly applies to analyze certain scenarios or case studies, and rarely talks about the digital perspective of Chinese ancient buildings, not to mention focusing on the characteristics of Chinese ancient buildings (especially its symmetry based on 3D laser scanning technology [19]). The related research work needs further exploration.

This paper is divided into five parts. The first part introduces the background and current situation of 3D laser scanning technology and symmetry research, followed by the determination of the research object and the descriptions of the research methods and data collection. The second to last part of the paper focuses on the case study of Heping Temple, analyzing the symmetry research. In the end, this paper will finish with a conclusion.

\section{Research Objects}

In this paper, 3D laser scanning technology is used to study the symmetry of Heping Temple in Beijing, exploring the intrinsic aesthetic value and cultural value in order to find a new way for the symmetry research of ancient buildings by using modern science and technology. The Heping Temple (Figure 1), located in Changping District of Beijing, is a Buddhist temple with a thousand-year history. According to the records of "Ri Xia Jiu Wen Kao", it stated that, on the Mount of Huata, there is a Heping Temple, which was built in Tang Dynasty under the supervision of Chigong Yu, a famous general in that era. The temple is named by Shimin Li, the emperor of Tang Dynasty, who wrote a letter to "Chici Heping Temple".

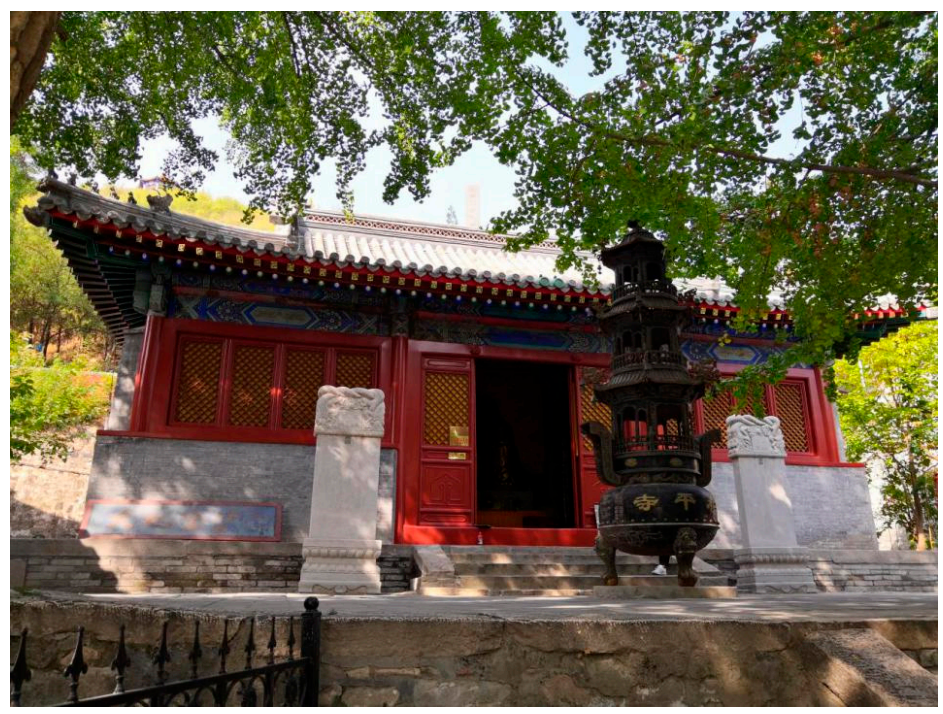

Figure 1. Current situation of the Heping Temple complex. 
The overall structure of the Heping Temple is a typical quadrangle courtyard. The main constructions include Tianwang Hall, the Grand Hall, Maitreya Hall and Guanyin Hall. Around the main buildings are the Reclining Buddha Hall, Apothecaries Hall, Wangsheng Hall and Yansheng Hall. The main axis of the architecture runs through the mountain gate, Tianwang Hall and the Grand Hall, highlighting their ritual status. The symmetrical layout with the core central axis also makes the Heping Temple complex orderly. Due to the chaos of war and disrepair, the integrity of the Heping Temple complex has been affected to some extent. The existing area is less than one fourth of that when it was first built, but the overall courtyard layout and the essential parts of the temple are still present, which provide an important support for our data collection and symmetry research.

\section{Methods and Data Collection}

\subsection{Methods}

3D laser scanning technology is used to survey ancient buildings, involving architecture, surveying and mapping, laser science and other disciplines in this paper. Architecture focuses on the history, art, construction techniques and protection of ancient buildings and the environment. Surveying and mapping study precision and accuracy. Laser science studies point cloud processing. This paper uses the method of literature analysis to quote, analyze and summarize the formed theoretical results as the basis of this research. The data acquisition method of the Heping Temple complex is studied in the form of case analysis, and the data acquisition scheme is designed. For the data that is not easy to investigate or is necessary to carry out experiments, the research is carried out through experimental methods (such as the experiment of thinning and point position error). Conclusions of some key parameters and performances are drawn, by doing experiments, to support the research results.

A three-dimensional laser scanner has two characteristics. One is the substation work, meaning that the data collected by each station are independent of each other. The second is fixed work, and slight vibration during work may lead to inaccurate data. These two characteristics are applied to ensure the accuracy of the collected data. When selecting a scanner, we mainly focus on two indicators of 3D laser scanner. One is its ranging capability (that is, its effective measuring area). Although data will also be collected out of this range, the accuracy is no longer guaranteed. The collected information can be deleted in post-processing. Another important parameter is the accuracy of the scanner, which is its ability to obtain information with precision. The accuracy is mainly related to the resolution of the scanner. The higher the resolution, the greater the amount of information obtained by the 3D scanner; therefore, the more comprehensive the content, the longer the data acquisition time. The ranging ability is often inversely proportional to the resolution. Generally speaking, the acquisition accuracy of 3D scanners with strong ranging ability is relatively low. The scanner with a high acquisition accuracy has a relatively weak ranging ability. We finally chose Faro's phase scanner over pulse scanner. This type of scanner can only collect information within $100 \mathrm{~m}$, but it is faster at data acquisition, more accurate in data processing, highly accurate and less erroneous. According to the size and characteristics of the Heping Temple complex, the phase scanner is more suitable.

As shown in Figure 2, this paper takes the point cloud image as the research basis, starting from the scale of ancient buildings through the method of point cloud measurement, which scans and collects the Heping Temple complex, splices the data, simplifies the model, and generates the point cloud image of the Heping Temple complex to ensure the accuracy and applicability of the research results. Then, the symmetry of ancient buildings is studied based on the point cloud image. 


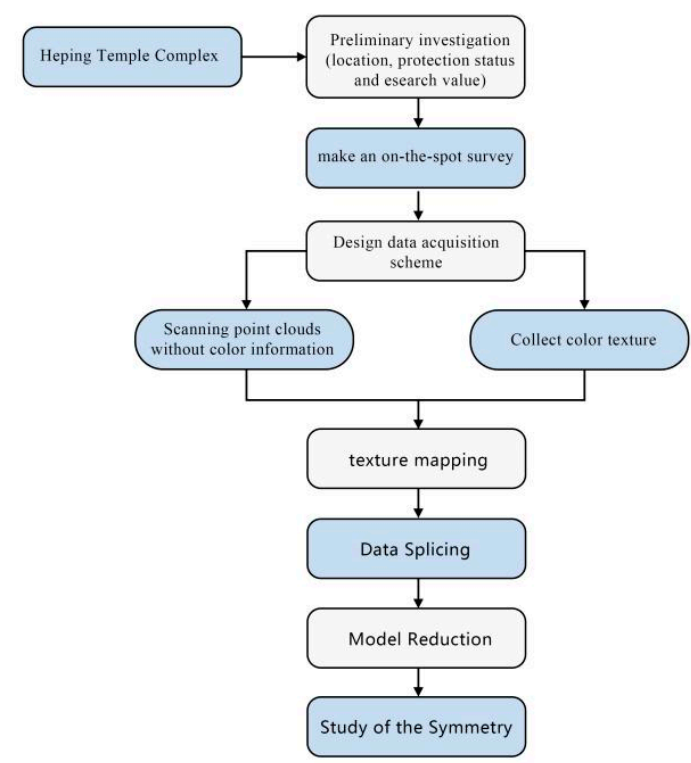

Figure 2. Research flow chart.

\subsection{Data Collection}

In the data acquisition stage of the Heping Temple complex, we used the equipment to carry out various measurements and acquisition work. This mainly includes control measurements, scanning layout measurement, point cloud data acquisition, texture image acquisition, field data inspection, data export backup, and so on [20].

Firstly, a controlled survey of the Heping Temple complex was carried out. The closed traverse was set through the control points, and the errors were averaged (also known as the process of adjustment) so a relatively accurate control network could be obtained and the quality of the overall data was ensured [21].

Secondly, a scanner was used to scan the Heping Temple complex in $360^{\circ}$ at different control points. [22]. There are three methods of layout survey (the uses of special points in space, control points, and the targets to form an organic whole in order to ensure that all sets of measured data are included in the same large coordinate system [23]). Thirdly, data collection of the Heping Temple complex was carried out. In other words, a scanner was used to scan the constructions of Heping Temple. At this time, the scanning system can be compared to a person's eyes. The scanner itself can be considered as a head, and the bracket can be treated as the body. The scanner captures what the human eyes can see. As shown in Figure 3, we used a 3D laser scanner to perform two $360^{\circ}$ scans at 42 different control points. Adhering to the principle of "control before measure, entirety before sections" during the process of data acquisition, low-resolution and low-quality data were gathered initially, and then the useful areas of the Heping Temple complex were scanned with high precision. Data acquisition was carried out at important sites, and then the missing details were scanned. Before starting, the direction of scanning should be determined, which is roughly the same the direction as the scanner when it first starts to work, facilitating manual splicing in the future. After collection, the data acquisition results of the Heping Temple complex should be tested in real time. At the same time, the relevant records should be made, and the errors should be supplemented for later data integration.

Finally, texture color information was gathered. After collecting the spatial information of the Heping Temple complex, the scanner will automatically take pictures to obtain the appearance information of the Heping Temple complex [24]. The ideal state is to scan when the weather is cloudy, so as to avoid the shaded area in dark or sunny days affecting the scanning results. The color cards can be used to correct after shooting, so the data quality can be improved [25]. 


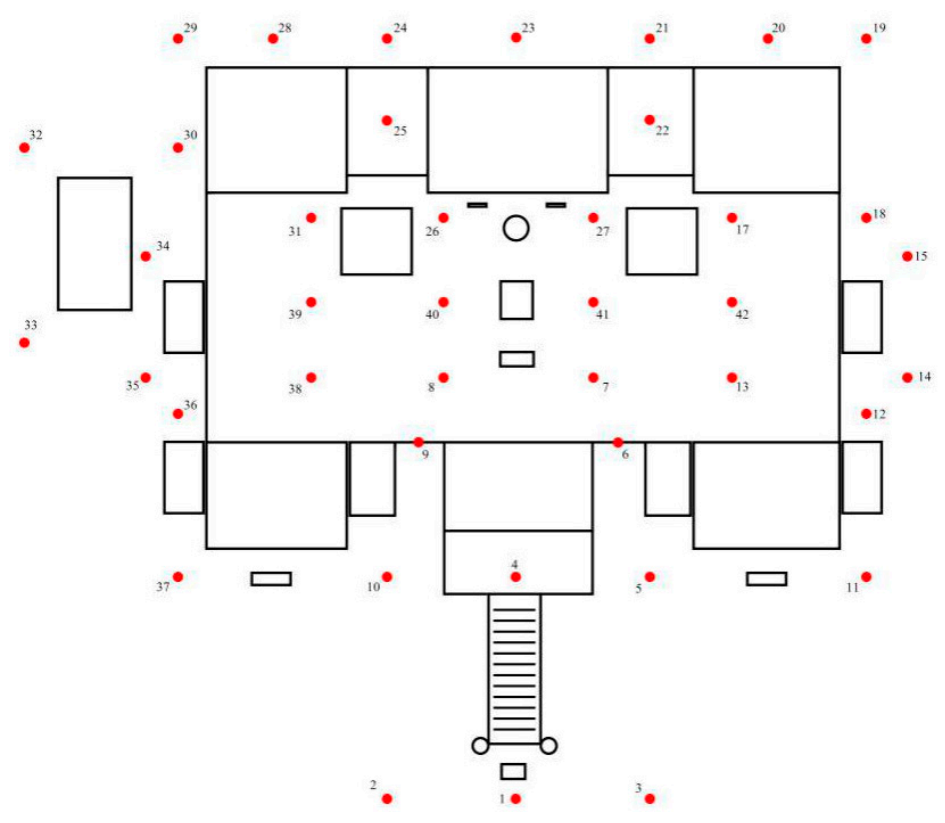

Figure 3. Schematic diagram of scanning positioning points of Heping Temple.

\subsection{Data Splicing}

In order to use the data in symmetry research, it is necessary to splice the original data into a 3D model, which requires coordinate integration of the original single station data and unifies all the data into one coordinate system [26]. In general, we will use geodetic coordinates to determine the spatial location information of the data. Therefore, we spliced all the data and transformed them into geodetic coordinates to realize the transformation from "relative orientation" to "absolute orientation" in geodetic coordinates. After data splicing, the collected data can be integrated into a point cloud model for subsequent research.

The data mosaic of the Heping Temple complex (Figure 4) is the process of merging the data of different sites into a unified coordinate system. The process of splicing is to use the principle of three points to determine a plane and align a surface with the 3D data collected by two stations. Then, the two stations will complete splicing and unifying to a coordinate system. The original data of single station have no relationship with each other, as they all adopt their own coordinate system (this is the inevitable result of sub-station data acquisition [27]). The splicing principle is shown in Figure 5. Assuming that the coordinate system o-xyz is the target coordinate system, in order to transform another coordinate system to the target coordinate system, it is necessary to translate along the three coordinate axes and rotate around the three coordinate axes, respectively. Because the 3D scanner is getting the actual size, the value of $m$ in the figure is zero. In order to realize the data splicing, three rotation parameters and three translation parameters need to be found. According to the knowledge of surveying and space geometry, three known non-collinear common points are needed to find these six parameters [28].

There are three methods to splice data: using the target, using control points, and using special points in space. First of all, when collecting data, targets are evenly arranged around the ancient buildings to be measured. The scanned target is equivalent to a point with coordinates. There are three identical points (or three identical targets) in the data of two stations, which can splice the data of two stations into a whole. By applying the second method, control points are used (also known as scanning on the control points). The coordinates of each control point can be obtained by using GPS, which is equivalent to the target and is the real geodetic coordinates. The method of control point layout for the Heping Temple complex is used to improve the measurement accuracy, as well as facilitate the data comparison and lay a solid foundation for the later data splicing. The last method 
is to use of special points in space. Three common obvious special points for splicing are manually selected. The first two methods have higher accuracy, but the third method lacks accuracy. However, when collecting large-scale data, too many targets will easily cause confusion and trouble.

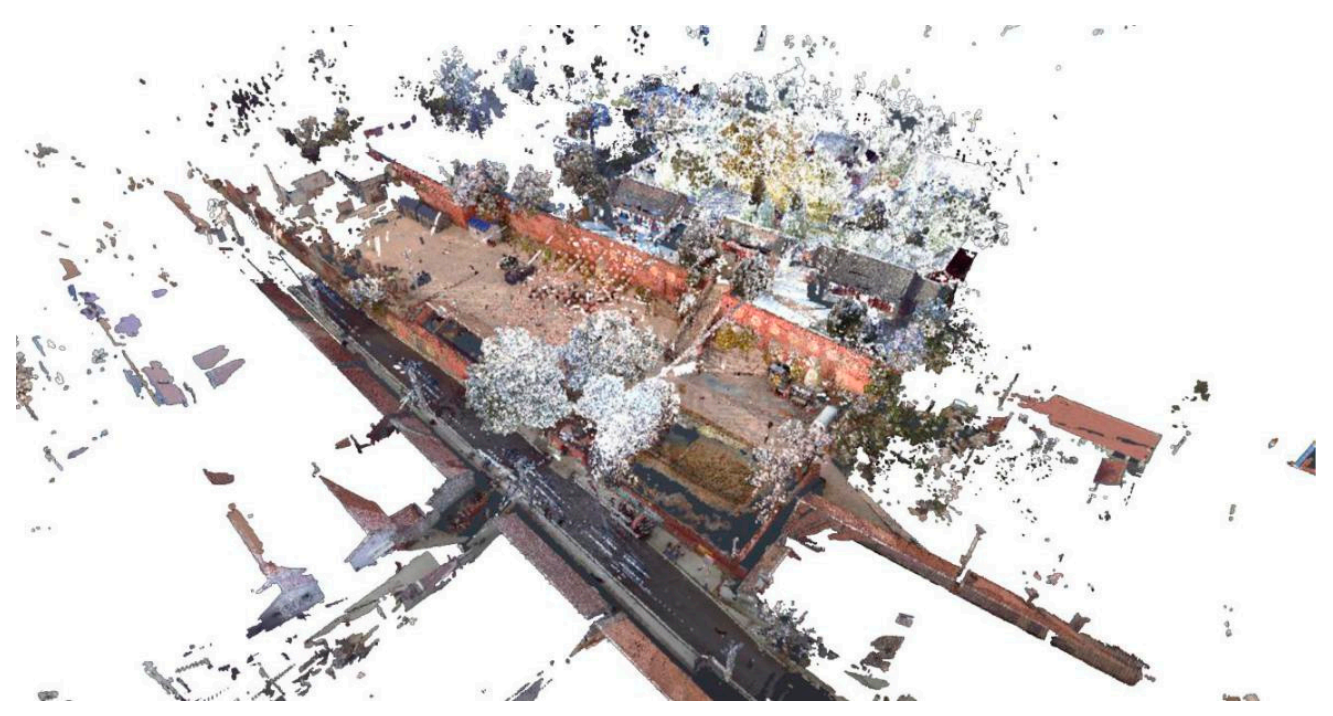

Figure 4. Mosaic results of point cloud model of the Heping Temple complex.

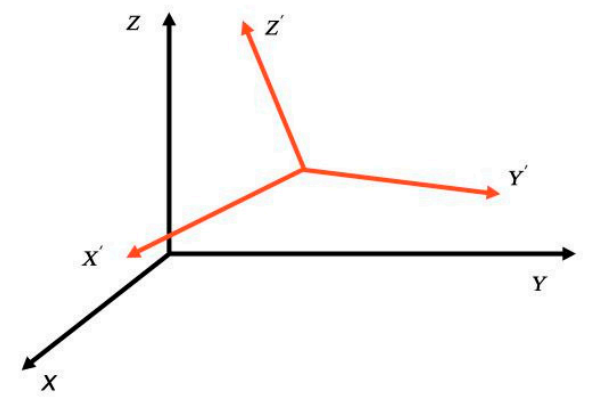

$$
\left[\begin{array}{c}
X^{\prime} \\
Y^{\prime} \\
Z^{\prime}
\end{array}\right]=(1+\mathrm{m})\left[\begin{array}{ccc}
1 & \varepsilon_{Z} & -\varepsilon_{Y} \\
-\varepsilon_{Z} & 1 & \varepsilon_{X} \\
\varepsilon_{Y} & -\varepsilon_{X} & 1
\end{array}\right]\left[\begin{array}{c}
X \\
Y \\
Z
\end{array}\right]+\left[\begin{array}{c}
\Delta X_{0} \\
\Delta Y_{o} \\
\Delta Z_{0}
\end{array}\right]
$$

Translation Parameter: $\Delta X_{0}, \Delta Y_{0}, \Delta Z_{0}$ Rotation Parameter: $\varepsilon_{X}, \varepsilon_{Y}, \varepsilon_{Z}$,

Scale Parameter: $\mathrm{m}$

Figure 5. Schematic diagram of model splicing.

\subsection{Model Reduction}

The data collection information of single station is limited, which cannot fully express the detailed information of the Heping Temple complex. Even in some key positions, there is no point cloud. For the requirements of registration and verification, there must be overlap between point clouds [29]. The scanner performed more than 82 scans. As the number of stations increases, the amount of information gathered begins to grow exponentially. The overlap of data caused the waste of point cloud and affected the computer's speed of reflection [30]. According to the principle of authenticity and integrity, the data of the Heping Temple complex need to be appropriately simplified on the premise of meeting the requirements to enhance its applicability [31].

There are three ways to simplify the data. The first method is to remove the noise, while the number of point clouds and the size of the file is also reduced. Another method is to simplify the data by thinning it according to a certain algorithm to reduce a certain amount of data. The third way is to import the data into other software programs (such as Geomagic), resample the point cloud and extract a certain amount of data according to a certain algorithm and then rasterize the data to transform the point cloud into a surface to reduce the size of the data.

In this paper, the Heping Temple complex is thinned. Under the premise that meets the accuracy requirements, the total number of points in the point cloud map of Heping Temple complex in proportion is reduced, so the aim of simplifying the data is achieved. 
In order to explore the most appropriate parameter setting of thinning [32], we take the Grand Hall of Heping Temple as an example. The limitation of thinning is that more thinning will affect the number of point clouds; less thinning is meaningless to reduce the size. The recommended numerical table of thinning ratio and maximum point spacing (Table 1) is obtained by reducing the total number of points in the point cloud map of the Grand Hall at each percentage and recording the density of the point cloud at its thinnest point. It should be noted that with the change of objective environment (such as distance between stations, incident angle, scanner accuracy, et cetera.), thinning parameters need to be adjusted accordingly.

Table 1. Recommended numerical table of thinning proportion and maximum point spacing.

\begin{tabular}{ccccccccccc}
\hline & \multicolumn{3}{c}{ Low Precision $(\mathbf{m m})$} & \multicolumn{2}{c}{ Medium Precision $(\mathbf{m m})$} & \multicolumn{3}{c}{ High Precision $(\mathbf{m m})$} \\
\hline Number of Stations & 2 & 3 & 4 & 2 & 3 & 4 & 2 & 3 & 4 \\
\hline Raw Data & 4.1 & 3.4 & 2.5 & 3.7 & 2.9 & 2.6 & 3.0 & 2.5 & 1.5 \\
\hline 10\% Thinning & 4.6 & 3.8 & 2.8 & 4.1 & 3.2 & 2.9 & 3.3 & 2.8 & 1.7 \\
\hline 15\% Thinning & 4.8 & 4.0 & 2.9 & 4.4 & 3.4 & 3.1 & 3.5 & 2.9 & 1.8 \\
\hline 20\% Thinning & 5.1 & 4.3 & 3.1 & 4.6 & 3.6 & 3.3 & 3.8 & 3.1 & 1.9 \\
\hline 25\% Thinning & 5.5 & 4.5 & 3.3 & 4.9 & 3.9 & 3.5 & 4.0 & 3.3 & 2.0 \\
\hline 30\% Thinning & 5.9 & 4.9 & 3.6 & 5.3 & 4.1 & 3.7 & 4.3 & 3.6 & 2.1 \\
\hline 35\% Thinning & 6.3 & 5.2 & 3.8 & 5.7 & 4.5 & 4.0 & 4.6 & 3.8 & 2.3 \\
\hline 40\% Thinning & 6.8 & 5.7 & 4.2 & 6.2 & 4.8 & 4.3 & 5.0 & 4.2 & 2.5 \\
\hline 45\% Thinning & 7.5 & 6.2 & 4.5 & 6.7 & 5.3 & 4.7 & 5.5 & 4.5 & 2.7 \\
\hline 50\% Thinning & 8.2 & 6.8 & 5.0 & 7.4 & 5.8 & 5.2 & 6.0 & 5.0 & 3.0 \\
\hline
\end{tabular}

\section{Results}

\subsection{Symmetry of Building Groups}

Based on the point cloud image of the Heping Temple complex, the middle axis space was measured and analyzed [11]. The central axis space of the Heping Temple complex is the core religious ritual space in the complex, which represents the order of Buddhist etiquette. From the perspective of the composition of the building group, the Heping Temple complex is symmetrically distributed along the central axis, and each building unit is arranged in an orderly manner. At the same time, the building base is gradually raised in accordance with the natural terrain, so that the unit with a higher level order in the building group is in the center of the composition and has a better vision, reflecting a strict cultural order and aesthetic representation.

\subsubsection{Axis Sequence of Plane Relation}

According to the measurement and analysis of the point cloud image, the Heping Temple complex is composed of $2.7 \mathrm{~m}$ high walls and buildings of different scales, and it is relatively closed. On the other hand, it forms an ample courtyard space. The gate, Tianwang Hall and the Grand Hall constitute the central axis of the plane layout of Heping Temple. The gate is located at the starting point of the axis sequence of Heping Temple. What is inside the gate is the first courtyard of Heping Temple. After 33 steps on the axis, it reaches Tianwang Hall. Behind Tianwang Hall is the main courtyard of Heping Temple, and the Grand Hall is located at the end of the axis. On the basis of the point cloud map of the Heping Temple complex, we marked the boundaries of different spaces and take the scale relationship between each space and the central axis as the basis for symmetry research [33]. The main axis from the mountain gate to the Grand Hall (Figure 6) controls the plane relationship of Heping Temple. The elevation difference and the turning of the path in the courtyard form the small nodes in the plane relationship, emphasizing the sense 
of sequence of the central axis of symmetry and ensuring the continuity of space on the axis sequence.
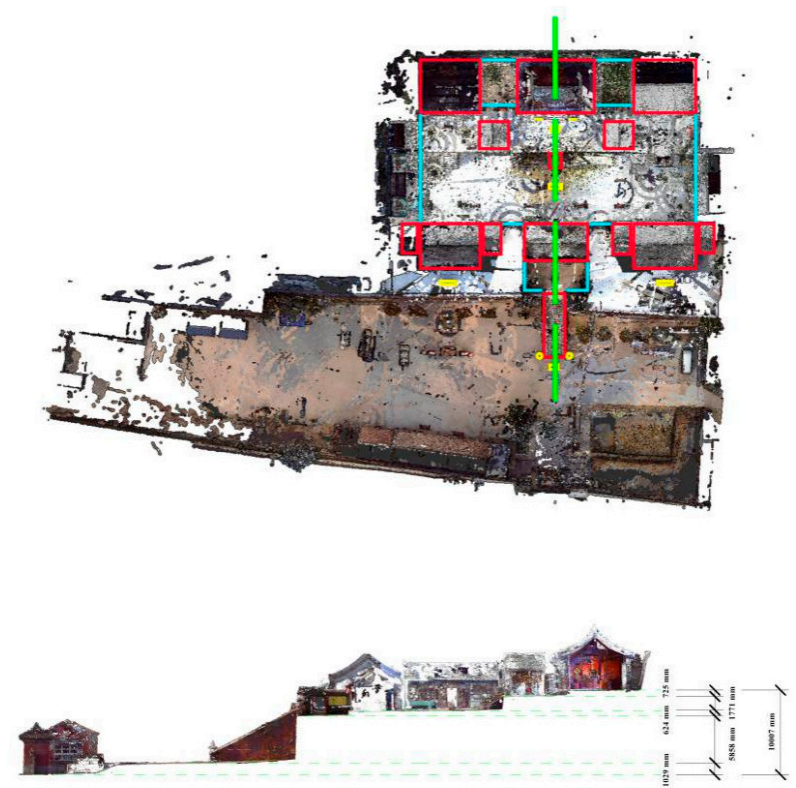

Figure 6. Space point cloud map of axis of Heping Temple building group.

\subsubsection{Symmetrical Layout of Spatial Organization}

The spatial organization of the Heping Temple complex (Figure 7) is characterized by a symmetrical layout. Whether it is a single courtyard surrounded by a group of buildings or a courtyard group connected by multiple courtyards, it shows its significant characteristic of symmetry. The Reclining Buddha Hall and the Apothecaries Hall are located on the East and West sides of the Tianwang Hall, respectively. The East and West sides of the Grand Hall are Guanyin Hall and Maitreya Hall. The Grand Hall connected by a small garden sized $6.75 \mathrm{~m} \times 6.75 \mathrm{~m}$. Tianwang Hall, the Grand Hall, and the surrounding auxiliary halls (together with the ancillary buildings) form a centripetal courtyard, showing a clear symmetrical layout. As a whole, it is fair to consider the Heping Temple complex symmetrical, and there are also individual asymmetric units arranged according to the natural terrain. For instance, there is no corresponding symmetrical unit in the Qihe Hall beside the Zushi Hall, and the first courtyard behind the gate conforms to the terrain in a trapezoidal shape. However, the reduction in local symmetry does not bring negative effects. On the contrary, it shows the flexibility of the symmetrical layout of Heping Temple.

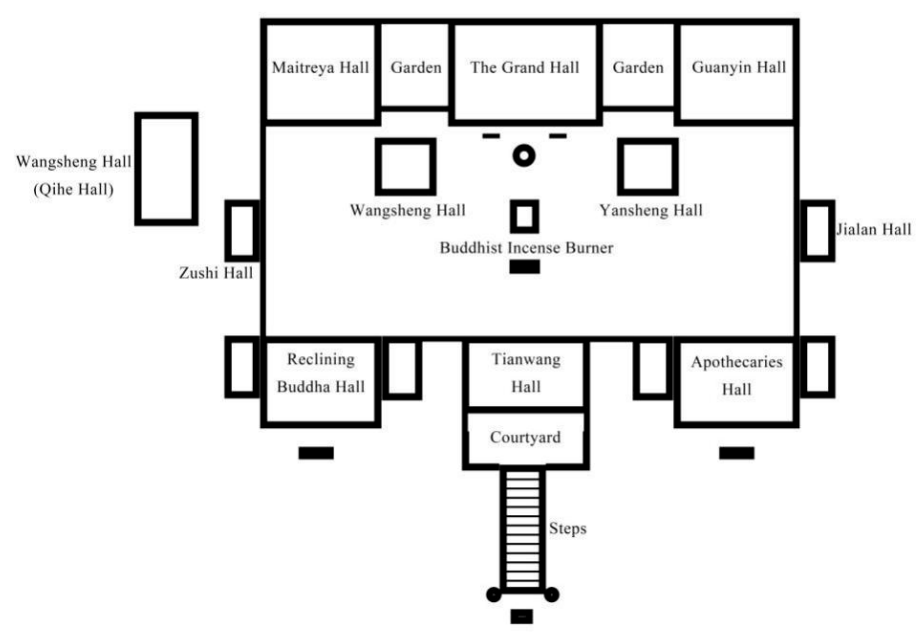

Figure 7. Space organization diagram of Heping Temple building group. 


\subsection{Symmetry of Individual Building}

Each and every individual building of Heping Temple embodies the characteristics of symmetry of ancient Chinese buildings and takes the central axis as the center to produce a variety of changes in the vertical direction. In addition, there is another axis of symmetry on the gable side of the building that also makes it symmetrical. This morphological feature basically follows the architectural form of ancient Chinese architecture [34]. The main building of the Heping Temple complex, the Grand Hall, adopts a clear way of construction: three bays, a gable and a hip roof with a single eave. We use hierarchical symmetry to classify and analyze the individual buildings. The individual buildings of the Heping Temple complex have two arrangements of symmetry. Both the plane layout and the facade form reflect the rigorous symmetry characteristics. Taking the Grand Hall as an example, it is located on the central axis of the Heping Temple complex, and its internal plane layout and facade form also show the characteristics of symmetry.

\subsubsection{Symmetry of Plane Layout}

In order to understand the symmetry of the plane layout of it in a better way, the Grand Hall point cloud image (Figure 8) was sliced and the wall boundary line was extracted for analysis [35]. According to the cross-section image at $0.5 \mathrm{~m}$ of the Grand Hall, the four corners of the walls of the Grand Hall are $90^{\circ}$ right angles. The North and South walls and the East and West walls are parallel to each other. The overall plane of the Grand Hall is a symmetrical rectangle. A cross-section at $1.5 \mathrm{~m}$ of the Grand Hall was also taken. From the same figure, it is illustrated that the wall expands outwards when the height rises to $1.5 \mathrm{~m}$, but the layout is still symmetrical. In addition, the figure also shows that the arrangement of the plane column network of the Grand Hall presents a unified symmetrical order. The spacing between the columns and the wall and the spacing between the columns are almost unified, showing a rigorous rational symmetrical aesthetic along the axis.

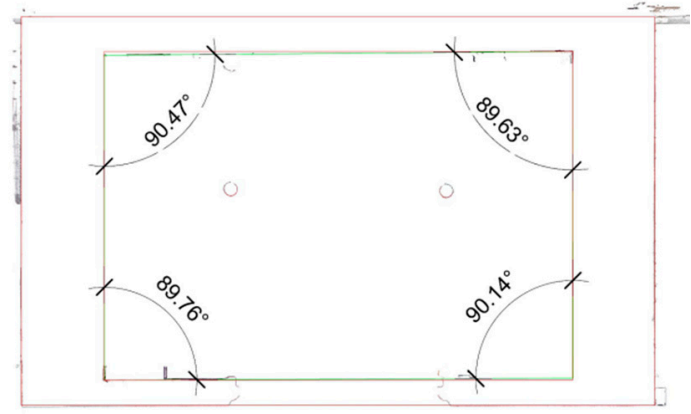

(a)

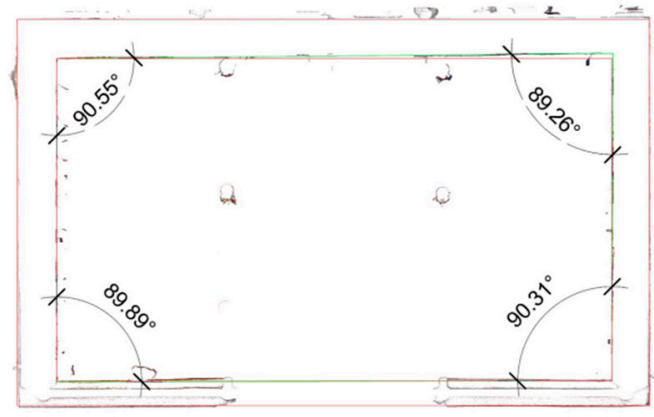

(b)

Figure 8. Horizontal section of the Grand Hall: (a) $0.5 \mathrm{~m}$ horizontal section; (b) $1.5 \mathrm{~m}$ horizontal section.

\subsubsection{Symmetry of Facade Form}

Through analysis of the 3D laser scanning elevation images of the Grand Hall (Figures 9 and 10) starting from the repeating texture and edge information [36,37], it is possible to determine the regularized features of the Grand Hall elevation shape through scale measurement in order to evaluate its symmetry [30]. As shown in the Figure 9, the South facade of the Grand Hall has three bays, showing a bilateral symmetry along the central axis. The width of the stairs at the entrance is $3798.2 \mathrm{~mm}$, which is the same as the width of the Middle Bay. Four lattice windows are arranged symmetrically in the left and right bays. A plaque is hung in the center of the forehead of the door, upon which is a gable and a hip roof. The distances between the East and West Gables and the symmetry axis is $6152.2 \mathrm{~mm}$ and $6161.4 \mathrm{~mm}$, respectively, which are about the same. 


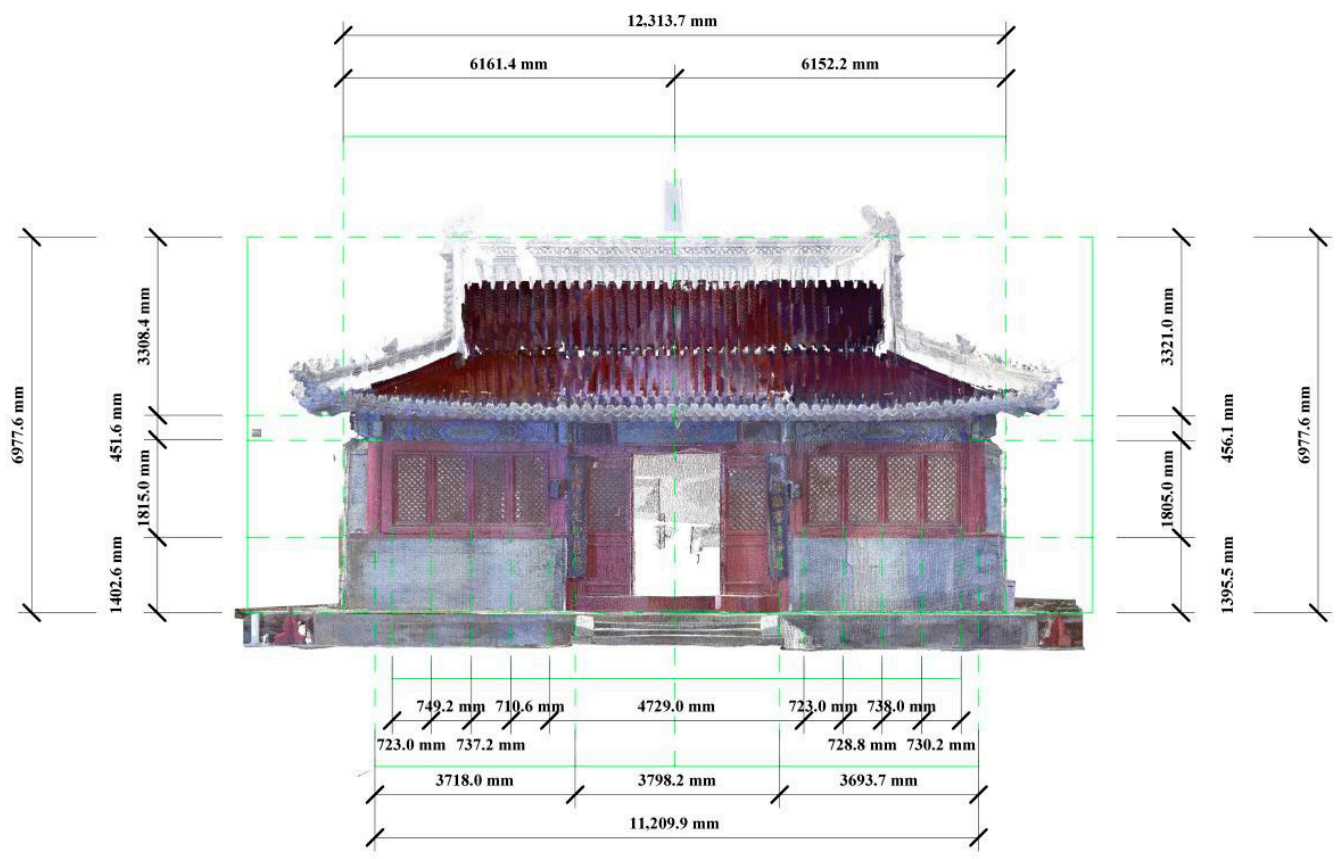

Figure 9. Point cloud picture of the South elevation of the Grand Hall.

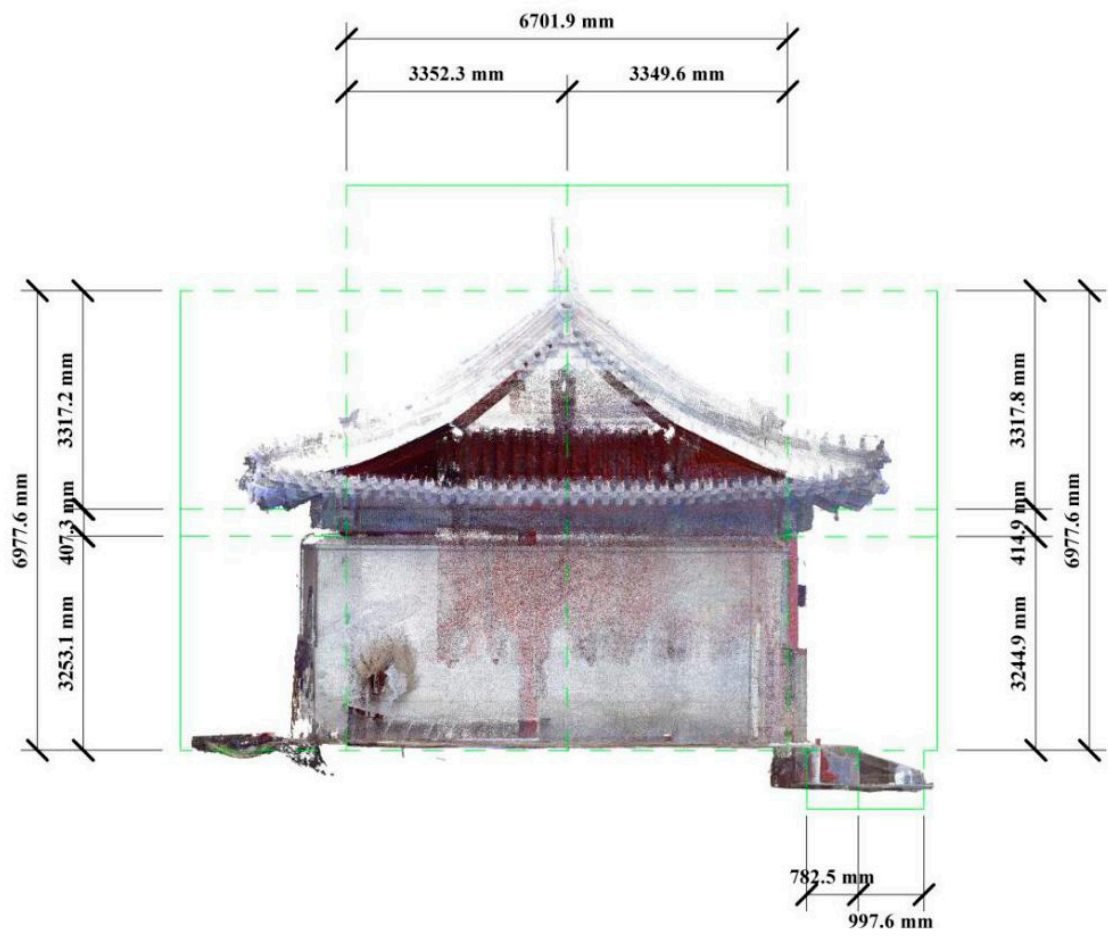

Figure 10. Point cloud picture of the East elevation of the Grand Hall.

In addition, we analyzed the local color and texture of the facade of the main hall through the Orthophoto Image of the color painting (Figure 11). By comparing the color paintings of architrave in the main hall, we found that the color and texture of architrave 1, architrave 2 and architrave 3 are axisymmetric, and architrave 1 and architrave 3 are also symmetrical, with the central axis of architrave 2 as the axis of symmetry. These colors and textures show symmetrical characteristics in scale, thickness, regularity and integrity. From the number of floors of the pedestal at the bottom, to the pattern and amount of pattern on doors and windows, to the animals at the top corner, there is no obvious difference between the primary and secondary points in the development of the vertical spatial sequence. They 
give visitors an intuitive cultural perception in the arrangement and volume. In general, the gable wall also tends to be axisymmetric; however, there are eaves in the North, so the northern wall is thicker than the southern wall in terms of visual effect. Therefore, we evaluate its symmetry by taking the center line of the overall structure as reference. The total width of the gabled roof is $6701.9 \mathrm{~mm}$. The facade shape is symmetrically distributed along the vertical line of the main ridge. However, as there is no entrance on the North side of the building, there are $997 \mathrm{~mm}$ long stairs in the South. In this way, in order to adapt to the terrain and arrangement of function, the overall view of the gable side will not be affected. As a result, the mountain wall can still achieve basic symmetry by visual perception.

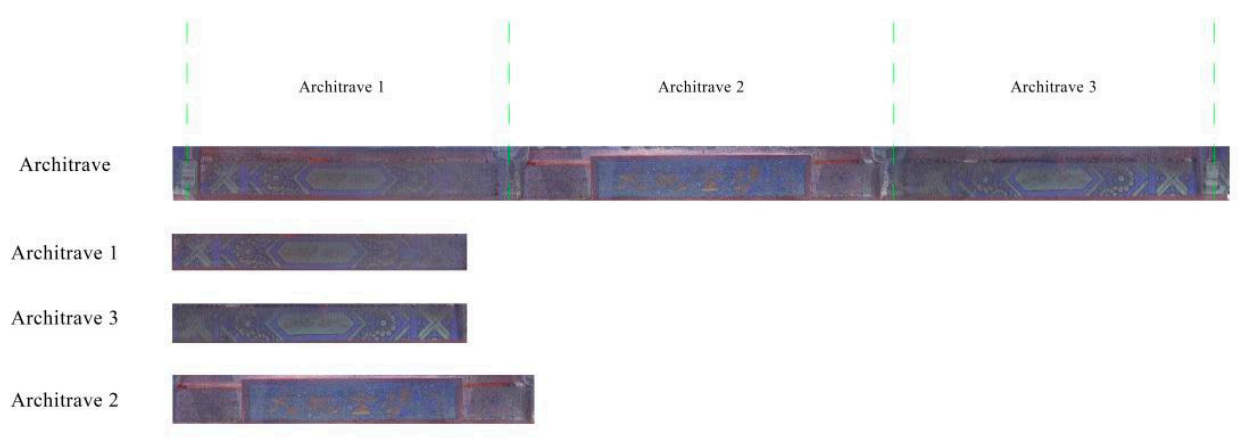

Figure 11. Orthophoto of the Architrave.

\subsection{Symmetry of Local Components of Buildings}

For the local components of Heping Temple, the doors and windows, brackets, stairs, ridges, tiles and other components are about symmetrical in shape, while the components (such as queti and ridgebeast) are asymmetrical in shape. However, they are arranged in pairs and symmetrically along the central axis. In a word, the local construction of Heping Temple reflects the symmetry in form and layout. In order to further analyze the symmetry of local components in Heping Temple, the building frames and purlins are chosen for high-precision symmetry performance analysis.

\subsubsection{Symmetry of Facade Form}

Taking the Grand Hall as an example, both the East and West beam frames are axially symmetric (Figures 12 and 13). Since the temple has been built for a long time, the supporting columns and beams of the frames have been deformed to various degrees [38]. The deformation of the support column is between $25-145 \mathrm{~mm}$, and its angle varies between $0.4^{\circ}$ and $3.5^{\circ}$. The malformation of the beams is between $25-55 \mathrm{~mm}$, and their angles vary between $0.4^{\circ}$ and $0.85^{\circ}$. The supporting column in the middle and the land subsidence (Figure 14) result in the difference in these deformations. However, these deviations are within the range of safety consideration and the overall frame is uniformly stressed. What's more, through the analysis of the ground settlement of the point cloud map of the Grand Hall, it is apparent that the ground settlement is symmetrical along the plane axis, profiting from the balanced symmetrical stress within the hall.

The symmetry of architectural framework is the external manifestation of structural form. This symmetrical structure plays an important role in the process of force transmission and support and constructs the internal functional space. The Grand Hall integrates the symmetry of internal structure with that of external form. It not only conforms to the structural characteristics of mechanics, but also achieves the aesthetic expression of each individual building, which comprehensively shows the cultural and aesthetic value of architecture. 


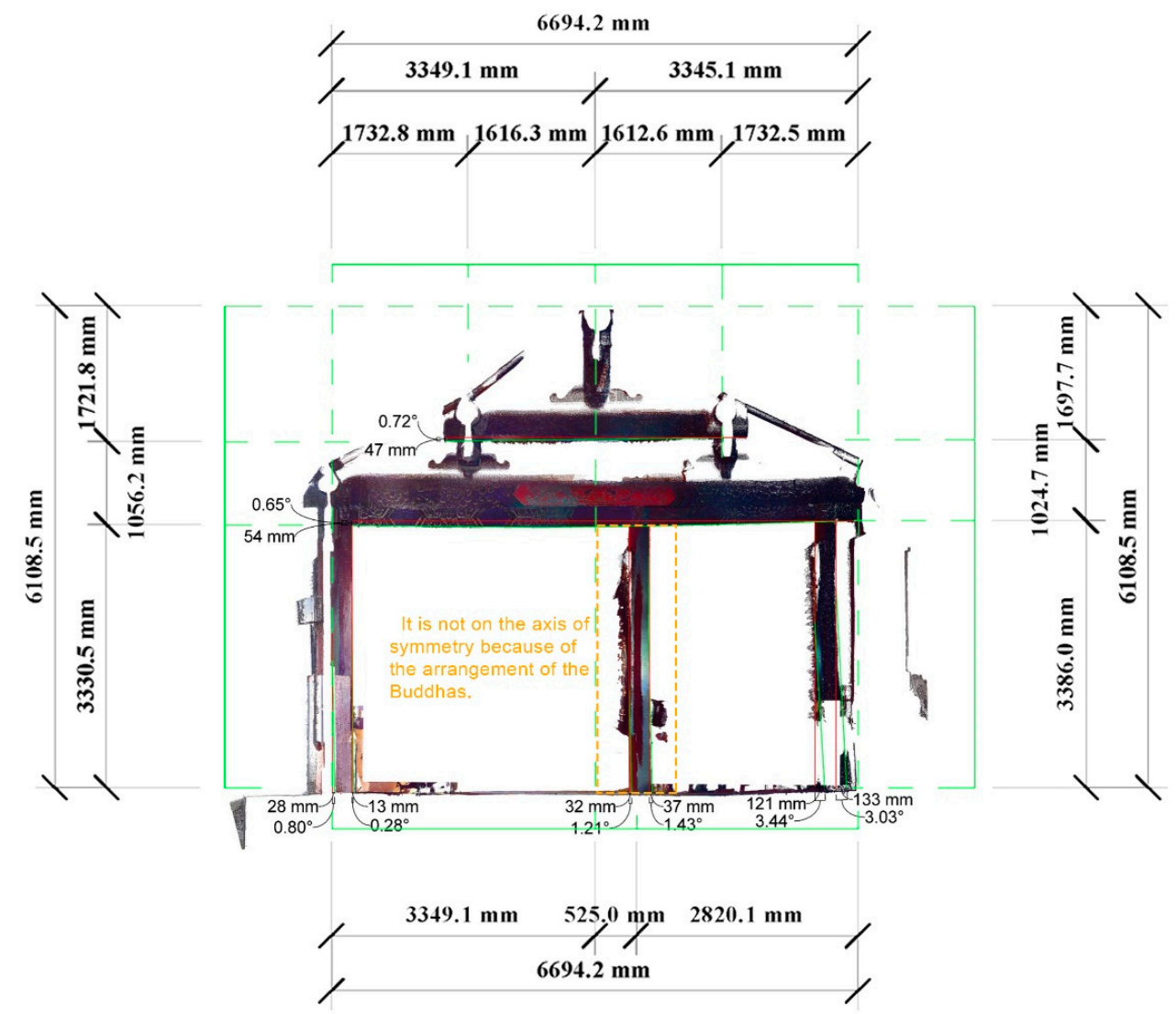

Figure 12. Point cloud picture of the beam frame section of the East seam.

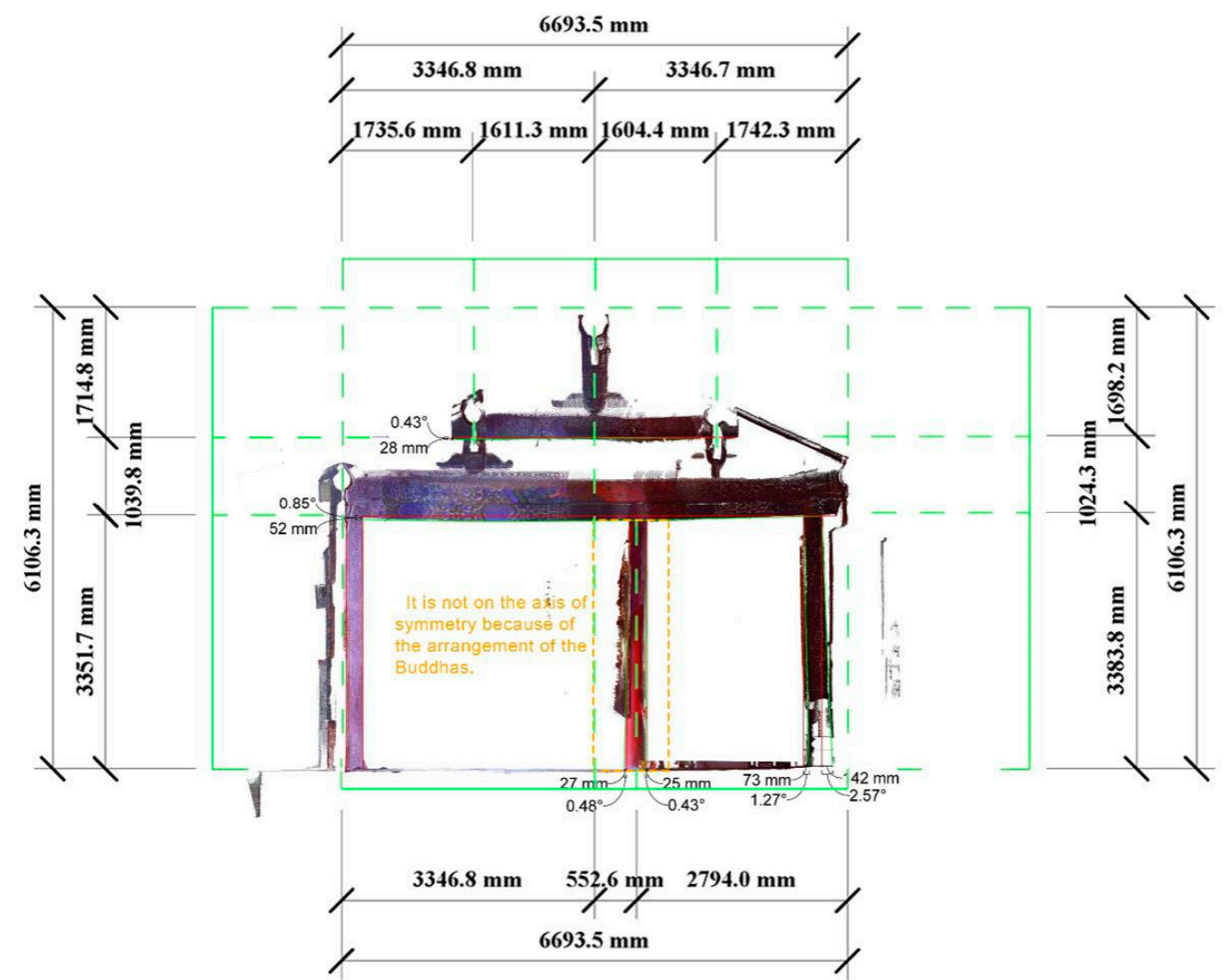

Figure 13. Point cloud picture of the beam frame section of the West seam. 

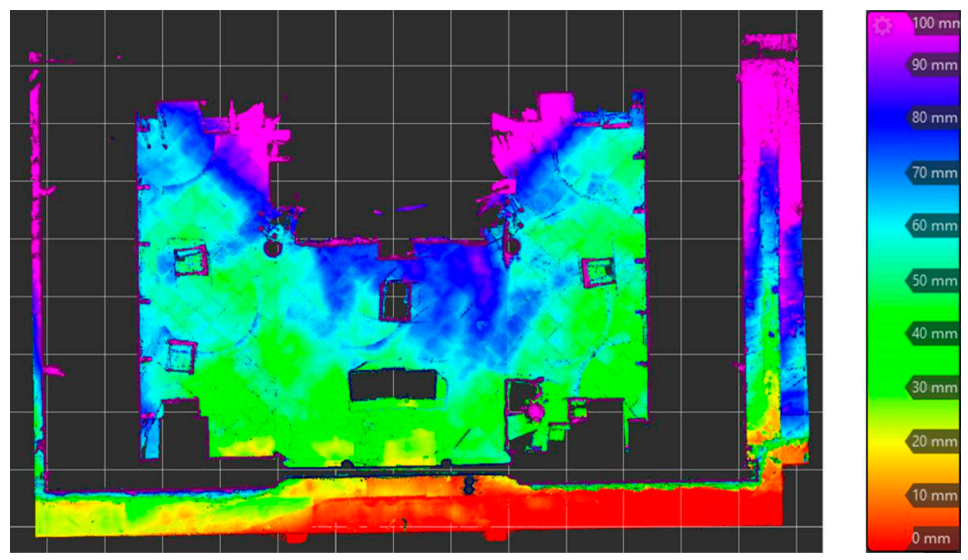

Figure 14. Analysis of point cloud of ground subsidence in the Grand Hall.

\subsubsection{Symmetry of Purlin}

In order to study the symmetry of purlins [39], each purlin of the Grand Hall is sliced (Figure 15) and numbered as 1-1 eaves purlin of front eaves, 2-2 intermediate purlin of front eaves, 3-3 ridge purlin, 4-4 intermediate purlin of rear eaves and 5-5 eaves purlin of rear eaves, respectively. It can be seen from the sectional diagram that each purlin has a deformation, the offset is between $10-50 \mathrm{~mm}$, and the offset angle is between $0.15^{\circ}$ and $0.9^{\circ}$. Among the five purlins, the deviation of the ridge purlin is relatively large, because it is in the highest position of the building and any structural dislocation below will cause the deviation of the back purlin. Even so, the offset of the ridge purlin is almost symmetrical, which is inseparable from the symmetrical design and component performance of the Grand Hall.

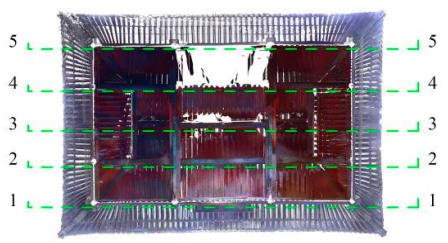

(a)

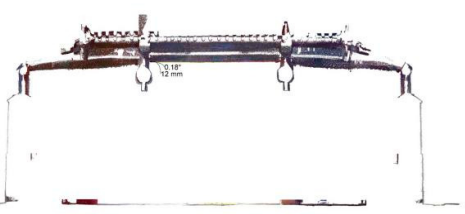

(c)

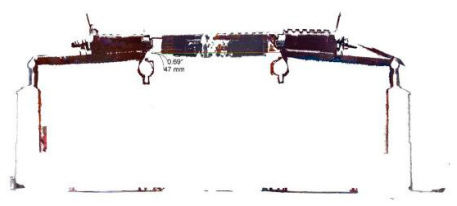

(e)

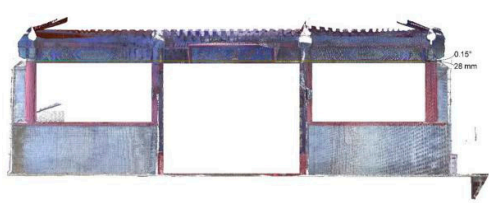

(b)

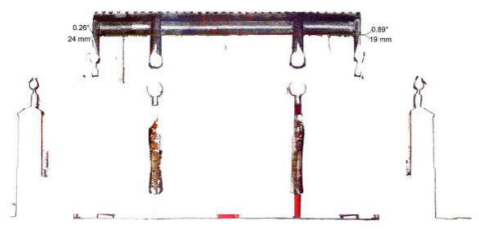

(d)

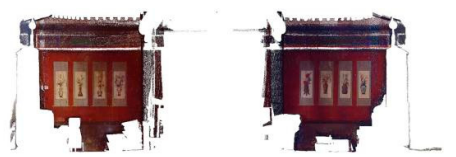

(f)

Figure 15. Point cloud diagram of purlin of the Grand Hall: (a) schematic diagram of cutting position, (b) 1-1 eaves purlin section of front eaves, (c) 2-2 intermediate purlin section of front eaves, (d) 3-3 ridge purlin section, (e) 4-4 intermediate purlin section of rear eaves, (f) 5-5 eaves purlin section of rear eaves. 


\section{Conclusions and Discuss}

Through the use of 3D laser scanning technology, it was shown that the Heping Temple complex in Beijing has symmetry characteristics in group layout, individual form and local components. From the perspective of architectural aesthetics, the Heping Temple complex takes the North-South central axis as the main line and symmetrically arranges each building unit. Each unit and its internal components are also placed in the form of a central axis of symmetry. This architectural space consciousness concerning the central axis is the internal expression of order and etiquette. From the perspective of culture and aesthetics, such frequent use of symmetry can be seen in the moderation culture and rigorous form of the aesthetics of Heping Temple. Chinese culture stresses the golden mean and harmony. The concept of "golden mean" is embodied in the ancient architecture; that is, the plane of the building is symmetrically and evenly arranged. There must be a solemn North-South central axis in the layout, which plays a central role. This pattern has become the epitome of the combination of Chinese ancient architecture, such as palaces, royal mansions, government offices, temples, ancestral halls, guild halls, academies, etc. Only when Chinese ancient architecture fully meets these requirements can it finally be synchronized with the Chinese people's traditional behavior mode of dealing with social interpersonal relations according to distance, age and distance, and then according to height, inferiority and nobility. Therefore, the frequent use of symmetry in Chinese ancient architecture is not only to symbolize eternity and to reflect the sense of order, but also to create a spatial sequence to connect the whole architectural group. In this way, a deep understanding of the essence of symmetry in ancient Chinese architecture is established, opening a door to reveal the aesthetic essence of ancient Chinese architecture.

Three-dimensional laser scanning technology has potential in the field of ancient architecture research. In this paper, 3D laser scanning technology is used to enhance people's understanding of the symmetry of the Heping Temple complex in order to deepen the understanding of the cultural connotation and aesthetic value of Chinese ancient architecture. The deepening research work continues.

Author Contributions: Author Contributions: Conceptualization, F.X. and D.S.; methodology, F.X.; software, F.X. and S.C.; validation, F.X.; formal analysis, F.X.; investigation, F.X.; supervision, F.X., S.C. and D.S.; visualization, S.C.; writing-original draft, F.X.; writing-review \& editing, D.S. All authors have read and agreed to the published version of the manuscript.

Funding: This research received no external funding.

Data Availability Statement: Data is contained within the article. The data presented in this study are available in Table 1.

Conflicts of Interest: The authors declare no conflict of interest.

\section{References}

1. Weyl, H. Symmetry; Princeton University Press: Princeton, NJ, USA, 2015.

2. Woods, H.J. 19-The geometrical basis of pattern design. Part I: Point and Line Symmetry in Simple Figures and Borders. J. Text. Inst. Trans. 1935, 26, T197-T210. [CrossRef]

3. Hubert, H.W. Symmetry and Symmetry: The Notion of the Antique Term Symmetria before Its New Definition in the Renaissance. Eur. Rev. 2021, 29, 210-225. [CrossRef]

4. Liang, S. Chinese Architecture: A Pictorial History; Dover Publications: New York, NY, USA, 2005.

5. Lundquist, J.M. The Temple: Meeting Place of Heaven and Earth; Thames and Hudson: London, UK, 1993.

6. Santagati, C.; Laura, I. 123D Catch: Efficiency, Accuracy, Constraints and Limitations in Architectural Heritage Field. Int. J. Herit. Digit. Era 2013, 2, 263-289. [CrossRef]

7. D'Apuzzo, N. State of the Art of the Methods for Static 3D Scanning of Partial or Full Human Body. In Proceedings of the Conference on 3D Modeling, Citeseer, Paris, France, 13-14 June 2006.

8. Chang, K.; Ding, P.; Luan, S.; Han, K.; Shi, J. Design of a Portable 3D Scanning Device. In Advancements in Mechatronics and Intelligent Robotics; Springer: Berlin/Heidelberg, Germany, 2021; pp. 485-491.

9. Daneshmand, M.; Helmi, A.; Avots, E.; Noroozi, F.; Alisinanoglu, F.; Arslan, H.S.; Gorbova, J.; Haamer, R.E.; Ozcinar, C.; Anbarjafari, G. 3d Scanning: A Comprehensive Survey. arXiv 2018, arXiv:1801.08863. 
10. Beraldin, J.A.; Picard, M.; El-Hakim, S.F.; Godin, G.; Valzano, V.; Bandiera, A. Combining 3D Technologies for Cultural Heritage Interpretation and Entertainment. In Proceedings of the SPIE-The International Society for Optical Engineering, San Jose, CA, USA, 16-20 January 2005. [CrossRef]

11. Remondino, F. Heritage Recording and 3D Modeling with Photogrammetry and 3D Scanning. Remote Sens. 2011, 3, 1104-1138. [CrossRef]

12. Levoy, M.; Pulli, K.; Curless, B.; Rusinkiewicz, S.; Koller, D.; Pereira, L.; Ginzton, M.; Anderson, S.; Davis, J.; Ginsberg, J.; et al. The Digital Michelangelo Project: 3D Scanning of Large Statues. In Proceedings of the 27th Annual Conference on Computer Graphics and Interactive Techniques, New Orleans, LA, USA, 23-28 July 2000; pp. 131-144.

13. Wang, M. Three-D Laser Scanning Technology Applied in Measuring the Historical Architectures of The Palace Museum. Palace Mus. J. 2011, 6, 143-156. (In Chinese)

14. Boehler, W.; Marbs, A. 3D Scanning and Photogrammetry for Heritage Recording: A Comparison. In Proceedings of the 12th Int. Conf. on Geoinformatics-Geospatial Information Research: Bridging the Pacific and Atlantic University of Gävle, Gävle, Sweden, 7-9 June 2004; pp. 291-298.

15. Wu, Y.; Hu, Y. Virtual Restoration on Cultural Relics: A Case Study of the Restoration of the Thousand-Hand Bodhisattva in Dazu Country. China Cult. Herit. Sci. Res. 2013, 3, 33-36.

16. Guidi, G.; Russo, M.; Ercoli, S.; Remondino, F.; Rizzi, A.; Menna, F. A Multi-Resolution Methodology for the 3D Modeling of Large and Complex Archeological Areas. Int. J. Archit. Comput. 2009, 7, 39-55. [CrossRef]

17. Angelini, M.G.; Costantino, D.; Milan, N. 3d and 2d Documentation and Visualization of Architectural Historic Heritage. In Proceedings of the XXIII International CIPA Symposium, Prague, Czech Republic, 12-16 September 2011.

18. Beijing Institute of Ancient Architecture. Technical Specification for 3D Information Collection of Cultural Relics Buildings; Beijing Municipal Bureau of Market Supervision; DB11/T 1796-2020 §; 2020. Available online: http:/ /wwj.beijing.gov.cn/ bjww/resource/cms/article/bjww_363732/620732/2019101815543226017.pdf (accessed on 24 December 2020).

19. Kersten, T.P.; Lindstaedt, M. Image-Based Low-Cost Systems for Automatic 3D Recording and Modelling of Archaeological Finds and Objects. In Proceedings of the Euro-Mediterranean Conference, Limassol, Cyprus, 29 October-3 November 2012; Springer: Berlin/Heidelberg, Germany, 2012; pp. 1-10.

20. Karasik, A.; Smilansky, U. 3D Scanning Technology as a Standard Archaeological Tool for Pottery Analysis: Practice and Theory. J. Archaeol. Sci. 2008, 35, 1148-1168. [CrossRef]

21. Costanzo, A.; Minasi, M.; Casula, G.; Musacchio, M.; Buongiorno, M.F. Combined Use of Terrestrial Laser Scanning and IR Thermography Applied to a Historical Building. Sensors 2015, 15, 194-213. [CrossRef] [PubMed]

22. Ioannidis, C.; Tsakiri, M. Laser Scanning and Photogrammetry for the Documentation of a Large Statue-Experiences in the Combined Use. In Proceedings of the CIPA XIX International Symposium, Antalya, Turkey, 30 September-4 October 2003; pp. 517-523.

23. La Mantia, M.; Lanfranchi, F. The Reverse Modelling Realized by Digital Scans and Fotomodellazione: Based on Post-Processing Compared Several Noteworthy Episodes Contained in the Basilical Complex of San Lorenzo Fuori Le Mura in Rome. In ISPRS Trento Workshop; Citeseer: Trento, Italy, 2011; Volume 152.

24. Szolomicki, J. Application of 3D Laser Scanning to Computer Model of Historic Buildings. In Proceedings of the World Congress on Engineering and Computer Science, San Francisco, CA, USA, 21-23 October 2015; Volume 2.

25. Brizzi, M. 3D Laser Scanning as a Tool for Conservation: The Experiences of the Herculaneum Conservation Project. In Proceedings of the 7th International Symposium on Virtual Reality, Archaeology and Cultural Heritage VAST, Nicosia, Cyprus, 30 October-4 November 2006.

26. Wachowiak, M.J.; Karas, B.V. 3D Scanning and Replication for Museum and Cultural Heritage Applications. J. Am. Inst. Conserv. 2009, 48, 141-158. [CrossRef]

27. Xiao, Y.H.; Zhan, Q.; Pang, Q. 3D Data Acquisition by Terrestrial Laser Scanning for Protection of Historical Buildings. In Proceedings of the 2007 International Conference on Wireless Communications, Networking and Mobile Computing, Shanghai, China, 21-25 September 2007; pp. 5971-5974.

28. Balestrini, E.F.D.; Ballarin, M.; Balletti, C.; Buttolo, V.; Gottardi, C.; Guerra, F.; Mander, S.; Pilot, L.; Vernier, P. Survey Methods for Earthquake Damages in the 'Camera Degli Sposi' of Mantegna (Mantova). ISPRS-Int. Arch. Photogramm. Remote Sens. Spat. Inf. Sci. 2013, XL-5/W2, 265-270.

29. Boehler, W.; Marbs, A. 3D Scanning Instruments. In Proceedings of the CIPA WG 6, Corfu, Greece, 1-2 September 2002 ; Volume 9.

30. Murphy, M.; McGovern, E.; Pavia, S. Historic Building Information Modelling (HBIM). Struct. Surv. 2009, 27, 311-327. [CrossRef]

31. Bornaz, L.; Rinaudo, F. Terrestrial Laser Scanner Data Processing. In Proceedings of the XXth ISPRS Congress, Istanbul, Turkey, 12-23 July 2004.

32. Guidi, G.; Beraldin, J.-A.; Ciofi, S.; Atzeni, C. Fusion of Range Camera and Photogrammetry: A Systematic Procedure for Improving 3-D Models Metric Accuracy. IEEE Trans. Syst. Man Cybern. Part B (Cybern.) 2003, 33, 667-676. [CrossRef] [PubMed]

33. Enrico, Q.; Clini, P.; Ripanti, M. Fast, Low Cost and Safe Methodology for the Assessment of the State of Conservation of Historical Buildings from 3D Laser Scanning: The Case Study of Santa Maria in Portonovo (Italy). J. Cult. Herit. 2017, 24, 175-183.

34. Wi, S.Y.; Sung, D.C.; Shin, W.J. A Study on Constructing Eave Curve of Part Chunyeo in the Three-Kan Hipped and Gable-Roofed Buddhist Temples. J. Archit. Hist. 2017, 26, 35-44. 
35. Castagnetti, C.; Dubbini, M.; Ricci, P.C.; Rivola, R.; Giannini, M.; Capra, A. Critical Issues and Key Points from the Survey to the Creation of the Historical Building Information Model: The Case of Santo Stefano Basilica. In Proceedings of the 1st International Conference on Geomatics and Restoration: Conservation of Cultural Heritage in the Digital Era, GeoRes, Florence, Italy, 22-24 May 2017; Volume 42, pp. 467-474.

36. Böhm, J. Multi-Image Fusion for Occlusion-Free Façade Texturing. Int. Arch. Photogramm. Remote Sens. Spat. Inf. Sci. 2004, 35, 867-872.

37. Balsa-Barreiro, J.; Fritsch, D. Generation of Visually Aesthetic and Detailed 3D Models of Historical Cities by Using Laser Scanning and Digital Photogrammetry. Digit. Appl. Archaeol. Cult. Herit. 2018, 8, 57-64. [CrossRef]

38. Stober, D.; Žarnić, R.; Penava, D.; Podmanicki, M.T.; Jurašević, R.V. Application of HBIM as a Research Tool for Historical Building Assessment. Civ. Eng. J. 2018, 4, 1565-1574. [CrossRef]

39. Zuo, L. Glossary of Chinese Architectural Terms. In Diversity in the Great Unity; University of Hawaii Press: Honolulu, HI, USA, 2019; pp. 171-182. 